\title{
Nano-crystallization: An Efficient Technology for Targeting Chemotherapeutic drugs
}

Furqan Muhammad Iqbal ${ }^{\text {a, b, c }}$, Florence Delie ${ }^{\text {a, b }}$

a Institute of Pharmaceutical Sciences of Western Switzerland, University of Geneva, Rue Michel-Servet 1, 1211 Geneva, Switzerland.Eric.allemann@unige.ch

${ }^{b}$.School of Pharmaceutical Sciences, University of Geneva, 1 Rue Michel-Servet 1211 Geneva, Switzerland

c Department of Pharmaceutics, Faculty of Pharmacy, Bahauddin Zakariya University, Multan, Punjab, Pakistan

\begin{abstract}
Presently, nano-crystallization is widely accepted for increasing the solubility and biological barrier permeability of poorly soluble drugs. It improves the bioavailability of therapeutic agents, increasing the effectiveness for treating diseased conditions, and could be safely administered by oral, parenteral, or transdermal routes. Drug nanocrystals are drug particles coated with a thin polymer layer to enhance their stability and could be decorated with ligands for active targeting. In addition, nanocrystals, due to their morphological properties, improve cell internalization. Therefore, passive targeting by high cellular uptake and retention in the mononuclear phagocyte system (MPS) may be expected. Drug nanocrystals are formulated by either top-down or bottom-up methods and could be scaled up for industrial manufacturing. In the past few decades, nanocrystal formulation has been increasingly studied to overcome the limitations of BCS Class II and IV chemotherapeutic agents. The study of cytotoxic effects of drug formulation on cell lines gives an insight for estimating its in-vivo biodistribution. This review highlights the role of morphology, stabilizer, and ligand conjugation on drug targeting and cellular uptake in cancer cells, as well as a brief discussion on nanocrystal production.
\end{abstract}

Key Words: Nano crystallization, Chemotherapeutic agents, Targeting, Morphology, Stabilizer, Cell lines

\section{Introduction}

Drug targeting, also called smart drug delivery design, is aimed to increase the drug concentration at the diseased cells, preventing the healthy tissues of the body from unwanted effects. Site-specific targeting of therapeutic agents is among the major drug delivery associated difficulties. However, in the past few decades, it has been made possible by exclusive research in nanomedicine to formulate nanodrug carriers targeted for curing tumors without affecting the normal tissues [1,2]. Passive targeting relies on physico-chemical properties of drugs and their interaction with biological medium while active drug targeting involves drugs to reach the targeted site by the mean of surface ligands and specific recognition. Conventional drug administration presents toxic side effects due to nonspecific biodistribution, whereas targeted drug delivery systems prolong and localize their interaction specifically with diseased tissues. The development of nanocarriers like polymeric nanoparticles, liposomes, and 
micelles is expected to resolve drug-related undesirable side effects. They can also target small molecules such as genes, peptides, and siRNAs to the desired site [3-6]. However, despite the desirable characteristics of nanocarriers, they usually have low drug loading capacity. Therefore, formulating pure nano-sized stabilized drug particles is an alternative to developing drug carriers to reach the site of action in optimum quantity directly $[7,8]$.

Pharmaceutical nanocrystals are nano-sized particles composed of $100 \%$ pure drug ranging from few nanometers to less than $1 \mu \mathrm{m}$ using the minimum quantity of excipients to favor their solubility and bioavailability. They possess appropriate saturation solubility and dissolution rate velocity due to high surface-to-volume ratio. Nanocrystallization techniques are being adopted for poorly soluble drugs belonging to class II and class IV Biopharmaceutical Classification System (BCS) $[9,10]$. The nanodrug particles could be prepared either by topdown or bottom-up methods. Different polymers or surfactants are physically used to stabilize them. Top-down techniques are wet milling, High-pressure homogenization, microfluidization, and spray drying, whereas nanoprecipitation is bottom-up technology $[11,12]$. These methods can efficiently produce the drug nanocrystals with smaller particle size with higher solubility and tumor cell permeation. Stabilizing agents are not only used to stabilize the nanocrystals but also play a significant role in cell internalization. A brief description of work by previous researchers on nanocrystals formulations of anticancer drugs of Class II and IV, formulations methods, stabilizers used, particle size, polydispersibility index, zetapotential and their cytotoxicity study on cell lines is indicated in table 1. The Nanocrystals have numerous advantages in pharmacokinetic parameters depending on particle size reduction, morphological characteristics, variation in crystal structure and anisotropy. The drug nanocrystals in suspension or lyophilized powder could be formulated and administered as oral, nasal, ocular, parenteral, and other dosage forms $[13,14]$. However, the drug-associated to polymeric nanoparticles sometimes exhibits low bioavailability due to entrapment in the matrix. In comparison, nanocrystals are actual nano-sized stable active agents which are efficiently distributed and by ligand modification, they could be transported to the target sites in body cells for different therapeutic effects [15].

Drug targeting and pharmacological effects of cytotoxic drug nanocrystals are determined by in vitro, ex vivo, and in vivo evaluation. The cellular uptake and intracellular dissolution of nanocrystals could be influenced by their morphology, concentration, incubation time, surface coating by stabilizers, and type of cell line. Morphological characteristics of nano-sized drug crystals play a significant role in uptake, distribution, and retention in tumor cells [16]. In addition, polymer stabilizers and ligand conjugation can also enhance nanocrystal targeting. This review will discuss the nano-crystallization techniques, factors affecting cellular uptake of nanocrystals in cancer cells and tissues and highlight the comparison of cytotoxicity of anticancer drugs with their nanocrystal formulations to evaluate the benefits of nano crystallization. 
Table 1. Nanoccrystal formulations of BCS Class II and IV cytotoxic drugs using different stabilizers, formulation methods, and evaluation

\begin{tabular}{|c|c|c|c|c|c|c|c|}
\hline $\begin{array}{l}\text { Drugs } \\
\text { (BCS } \\
\text { class) }\end{array}$ & Stabilizers & $\begin{array}{l}\text { Nano } \\
\text { crystallization } \\
\text { Technique }\end{array}$ & $\begin{array}{l}\text { Particle } \\
\text { size } \\
\text { Range } \\
(\mathrm{nm}) \\
\end{array}$ & $\begin{array}{l}\text { PDI } \\
\text { Range }\end{array}$ & $\begin{array}{l}\text { Zeta } \\
\text { potential } \\
\quad(\mathrm{mV})\end{array}$ & Cell lines & Ref. \\
\hline \multirow[t]{3}{*}{$\begin{array}{l}\text { Paclitaxel } \\
\text { (IV) }\end{array}$} & $\begin{array}{l}\text { Tween-80, } \\
\text { Glycol Chitosan } \\
(\mathrm{GC}), \\
\text { sodium } \\
\text { (SA) alginate }\end{array}$ & Microfluidizer & $194-382$ & $\begin{array}{l}0.134- \\
0.194\end{array}$ & -18 to -36 & $\begin{array}{l}\text { Cancer cell line MCF7 } \\
\text { and MDA-MB }\end{array}$ & [17] \\
\hline & $\begin{array}{l}\text { Hyaluronic acid } \\
\text { Transferrin }\end{array}$ & Nanoprecipitation & $236-339$ & $\begin{array}{l}0.14- \\
0.21\end{array}$ & -2.7 to -16.6 & $\begin{array}{l}\text { MCF-7 and normal cell- } \\
\text { line, HaCaT cells }\end{array}$ & {$[18]$} \\
\hline & $\begin{array}{l}\text { D- } \alpha \text {-Tocopheryl } \\
\text { polyethylene glycol } \\
1000 \quad \text { succinate } \\
\text { (TPGS) }\end{array}$ & Co-precipitation & & & & $\mathrm{KB}$ and $\mathrm{H} 460$ cell lines & [19] \\
\hline \multirow[t]{2}{*}{$\begin{array}{l}\text { Campothecin } \\
\text { (IV) }\end{array}$} & $\begin{array}{l}\text { Hyaluronic acid } \\
\text { (HA) }\end{array}$ & $\begin{array}{l}\text { Nanoprecipitation } \\
\text { by Probe Sonicator }\end{array}$ & $196-400$ & $\begin{array}{l}0.19 \text { to } \\
0.223\end{array}$ & -4 to -22 & $\begin{array}{l}\text { CD44 Positive Cancer } \\
\text { Cells }\end{array}$ & [20] \\
\hline & boric acid & $\begin{array}{l}\text { Nanoprecipitation } \\
\text { by Probe Sonicator }\end{array}$ & 204- 496 & $\begin{array}{l}0.172- \\
0.586\end{array}$ & $\begin{array}{l}-5.82 \text { to }- \\
40.1\end{array}$ & $\begin{array}{l}\text { Schwann SC cells w, } \\
\text { human lung carcinoma } \\
\text { A549 cells, human } \\
\text { cervical carcinoma Hela } \\
\text { cells }\end{array}$ & [21] \\
\hline \multirow[t]{2}{*}{$\begin{array}{l}\text { Docetaxel } \\
\text { (IV) }\end{array}$} & Tween 80 & $\begin{array}{l}\text { Nanoprecipitation } \\
\text { by Probe Sonicator }\end{array}$ & $526-543$ & $\begin{array}{l}0.18- \\
0.24 \\
\end{array}$ & $\begin{array}{ll} & -9.6 \quad \text { to } \\
+10.1 & \\
\end{array}$ & $\begin{array}{l}\text { MCF-7, MDA-MB-231, } \\
\text { A549 and HaCaT cells }\end{array}$ & [22] \\
\hline & Transferrin & $\begin{array}{l}\text { Nanoprecipitation } \\
\text {,by Probe Sonicator }\end{array}$ & $405-468$ & $\begin{array}{l}0.18- \\
0.23\end{array}$ & -15 to -18 & A549 cells by MTT assay & [23] \\
\hline $\begin{array}{l}\text { Etoposide } \\
\text { (IV) }\end{array}$ & Pluronic F-127 & $\begin{array}{l}\text { Antisolvent } \\
\text { precipitation }\end{array}$ & $117 \pm 28$ & $\begin{array}{l}0.12- \\
0.13\end{array}$ & -16 to -20 & $\begin{array}{l}\text { CT26 and 3LL } \\
\text { cancer cells }\end{array}$ & [24] \\
\hline Sorafenib (II) & Polaxamer 407 & $\begin{array}{l}\text { High pressure } \\
\text { homogenization }\end{array}$ & $141-544$ & $\begin{array}{l}0.188- \\
0.797\end{array}$ & -7 to -28 & $\begin{array}{l}\text { HepG2 } \\
\text { cells }\end{array}$ & {$[25]$} \\
\hline Oridonin (IV) & $\begin{array}{l}\text { Polyvinyl } \\
\text { pyrrolidone } \\
\text { (PVP K30) }\end{array}$ & $\begin{array}{l}\text { Antisolvent } \\
\text { Probe sonication }\end{array}$ & $285-295$ & $\begin{array}{l}0.24- \\
0.27\end{array}$ & $\begin{array}{l}0.035- \\
0.085\end{array}$ & MDCK cells & [26] \\
\hline $\begin{array}{l}\text { Parthenolide } \\
\text { (IV) }\end{array}$ & F68 and lecithin & $\begin{array}{l}\text { High-pressure } \\
\text { homogenization } \\
\text { method (PHPH) }\end{array}$ & $126.9 \pm 2.31$ & $\begin{array}{l}0.230 \pm \\
0.024\end{array}$ & $\begin{array}{l}-11.18 \quad \pm \\
0.59\end{array}$ & HepG2 cell lines & [27] \\
\hline
\end{tabular}

*BCS: Biopharmaceutical classification system

\section{Nanocrystallization Techniques}

Pharmaceutical nanocrystals mainly consist of therapeutic ingredients with the minimum quantity of excipients for stabilization and cellular uptake. The simple nanocrystal engineering technologies and lesser ingredient requirements save the production cost. Therefore, nanocrystals could be easily scaled up for industrial manufacturing. Furthermore, nanocrystals could be conveniently sterilized by physical sterilization methods like dry heat, steam, cold filtration, and radiation, depending on the drug stability. The importance of nanocrystal sterilization has been evaluated by researchers in formulating nanocrystals of cytotoxic active 
ingredients $[28,29]$. Top-down and bottom-up techniques are used for nanocrystal synthesis. Both methods are illustrated in figure 1. In some situations, to obtain further size reduction and uniformity, the combination of these two methods could be adopted.

\subsection{Top-down Method}

Top- down methods involve high energy to disintegrate the drug to obtain nano-sized particles, either by wet milling or high-pressure homogenization technologies. These technologies have a high percentage yield that makes them suitable for large-scale manufacturing compared to bottom-up methods. Wet milling, specifically wet bead milling, involves the size reduction of usually poorly water-soluble drugs suspended in water with a nominal quantity of stabilizer by high energy forces in a tube/ chamber containing inert grinding media. The griding media could be either metallic (carbon steel, chrome steel, or stainless steel) or nonmetallic (alumina, zirconium oxide, glass etc), ranging from 0.05 to $2 \mathrm{~mm}$ in size. The particle size reduction mechanism mainly involves impact, high shear forces, and attrition generated by friction among particles. Wet bead milling is usually preferred to high-pressure homogenization due to particle size uniformity, easy scale-up and convenience in handling wide range of drug quantities [30-32].

However, high-pressure homogenization is the second well adopted top-down technology using high pressure for particle size reduction in liquid media in the presence of surfactants via particle collision, shear forces, and cavitation forces. Piston-Gap homogenization and micro fluidization (Jet-Stream Homogenization) are the two main homogenization procedures. The advantages of high-pressure homogenization are aqueous free preparation of nanocrystals and aseptic production for parenteral administration [33-35]. Particle size reduction and PDI of nanocrystals prepared by high pressure homogenization depends on homogenization pressure. In a research study, parthenolide nanocrystals were successfully prepared by high pressure homogenization using lecithin and F68 were employed as the stabilizers. The stabilizers rapidly covered the nanocrystal surface during homogenization, providing electrostatic and steric stabilization for efficient particle size reduction. The particle size reduction was mainly related to homogenization pressure i.e., by increasing from 500 bar to 1000 bar reduced the particle size from $208.2 \mathrm{~nm}$ to $126.9 \mathrm{~nm}$ and the PDI decreased from 0.333 to 0.230 [27].

Particle size reduction by top-down methods also depends on the physical properties of the active ingredient as it could be affected by factors like initial particle size, polymorphism, morphology, and young modulus [36-40]. Young modulus determines the strength, elastic, and plastic deformation of solid materials. Some specialized processes like freeze-drying and spray drying to modify drugs with characteristics suited for top-down procedures. Drug particles obtained with porous structures with reduced crystallinity after treatment by freezedrying or spray drying have an irregular molecular arrangement compared to unmodified crystalline particles. Due to porosity and brittleness, they have more breakage points and are well adapted for particle size reduction by high impact, shear, and attrition forces. For example, the optimized particle size of glibenclamide was obtained from amorphous freeze-dried powder after treatment by high-pressure homogenization [41]. In another research, resveratrol pretreated by spray drying resulted in nanosuspensions with significantly smaller particle sizes without reduction in crystallinity [42].

\subsection{Bottom-up Method}

Bottom-up technology, also named nanoprecipitation is based on solubilizing the drug in a solvent, followed by precipitation by the addition of non-solvent containing a stabilizer [43]. Nanoprecipitation method was first introduced by List and Sucker in 1988 [44]. Crystal growth 
is generated by nucleation occurring by either solvent-antisolvent mixing or solvent removal. The blending of drug solution and antisolvent could be done by magnetic stirring or impeller blades. It is followed by sonication to promote nucleation, the process known as nanoprecipitation. Optimization of the process variables and formulation aspects of nanoprecipitation is required to obtain the desired nanocrystal formulations. It primarily depends on the physical properties and concentration of the drug and, to a lesser extent, the stabilizing agents. Processing parameters influencing the final product are stirring speed, the ratio of solvent-antisolvent, type and depth of the sonicator's probe and temperature [45-47]. Using organic solvent for drug solubilization has led to limiting applications of bottom-up techniques. This is because of the lower solubility of some new therapeutic agents in easily removable organic solvents like acetone, ethanol, methanol etc., but soluble in dimethyl sulfoxide (DMSO). DMSO itself is toxic and removal consumes time that increases the overall production cost [48].

With the advancement in pharmaceutical technology, supercritical antisolvent precipitation using supercritical fluids could be used in nanocrystal production. Supercritical fluids behave like liquids and convert to gas above their critical point e.g., carbon dioxide, ammonia, ethane, ethylene etc [49]. The drug in a solvent is sprayed on supercritical antisolvent in a chamber, resulting in rapid nucleation and nanocrystal formation. This technique yields smaller particle size, uniformity at ambient temperature suitable for thermosensitive materials. Another technology for nanocrystal production is spray flash evaporation system is well adapted for many benefits of short processing time, particle size and low temperature. The drug dissolved in low boiling point solvents like acetone, ethanol or methanol at a concentration of 1 to $10 \% \mathrm{w} / \mathrm{v}$, under high pressure of 40 bars is introduced to the vacuum chamber. The pressure drop creates instant evaporation and crystallization [50].

\subsection{Combination Technologies}

Both top-down and bottom-up approaches for nanocrystal production have limitations in terms of longer running times of energy equipment or initial pretreatment to obtain micronized drug particles (51). To attain the specific benefits and minimize the disadvantages of these two approaches, combinative technologies are adopted. One commonly used combination technology is NANOEDGE ${ }^{\mathrm{TM}}$ introduced by Baxter (Baxter Pharmaceutical Solutions, LLC, US). It involves a pretreatment for microprecipitation of crystals, usually followed by high energy homogenization. The micro precipitation step decreases energy cost for running high shear equipment, whereas subsequent treatment by high-pressure homogenizer increases size uniformity and thermodynamic stability of nanocrystals [52, 53]. Other combination approaches are H42 and H96 technologies involving a pretreatment by spray drying and lyophilization (freeze-drying) followed by high-pressure homogenization. Both sprays drying and lyophilization remove excess moisture content from drug particles, making them more brittle/breakable by high-pressure homogenization. With freeze-drying, H96 technology is more suitable for thermosensitive drugs $[54,55]$.

Some combination technologies combine the two top-down techniques, like wet ball milling and high-pressure homogenization. First, the drug particle suspension is subjected to low energy ball milling, with conditions adjusted to yield a particle size in sub-micron range from $600 \mathrm{~nm}$ to 1 micron or slightly bigger, then treated by high-pressure homogenization. The pretreatment with ball milling reduces the overall cost and saves time and energy by a highpressure homogenizer. Moreover, this method ultimately yields particles with better stability and uniformity but relatively larger particle size compared to the above-mentioned combination technologies $[56,57]$. 


\section{Top- down technique}
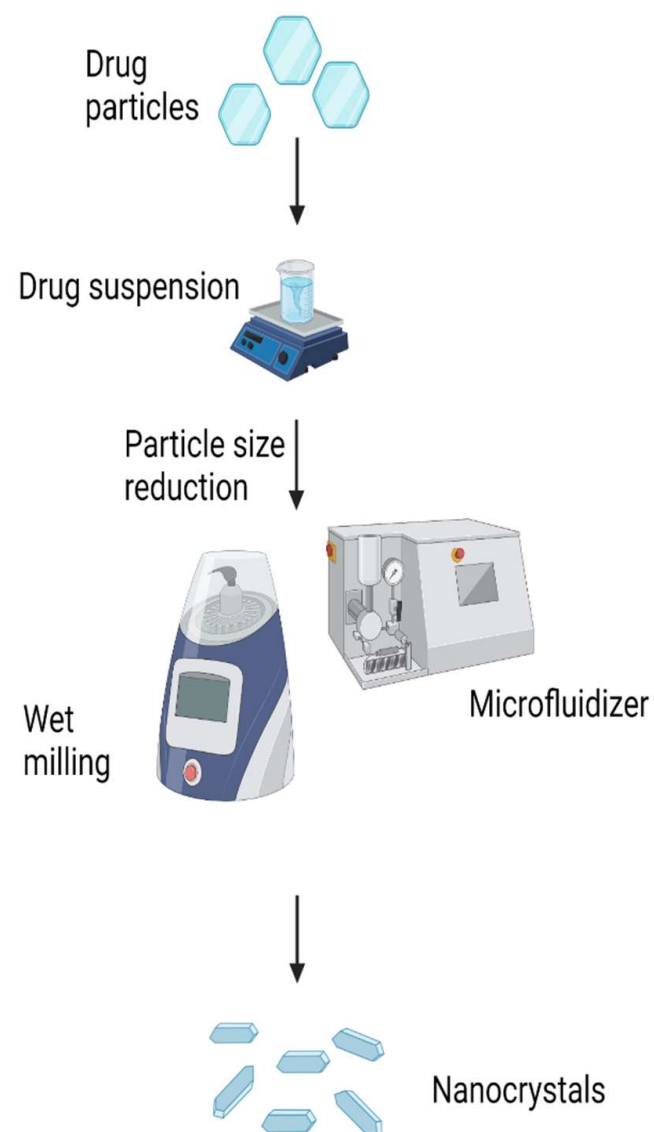

$\Leftrightarrow$ Nanocrystals
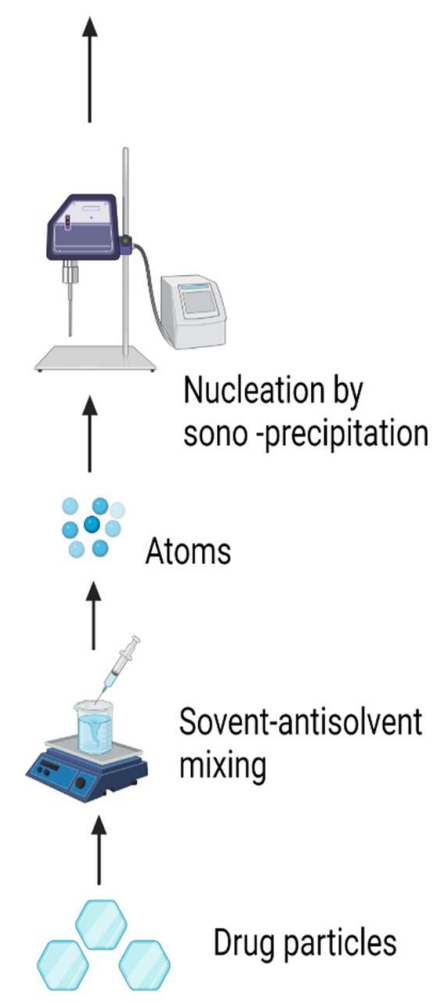

Bottom-up technique

Figure 1. Schematic illustration of nanocrystal production methods, showing general processing of top-down approach and bottom-up technologies (created with BioRender)

\section{Cellular uptake of Nanocrystals}

The presence of receptors has a significant role in cell permeation of few drugs and their cellular uptake. However, numerous drugs have remarkable therapeutic effects but poor permeation characteristics and cellular uptake. These limitations could be overcome by drug nanocrystal modification and formulation techniques to enhance bioavailability and increase cell internalization. Furthermore, the internalization of nanocrystals could be influenced by morphological characteristics, type of stabilizer used to coat the nanocrystal surface, and ligand attachment specific to infectious cells, as illustrated in figure 2. 


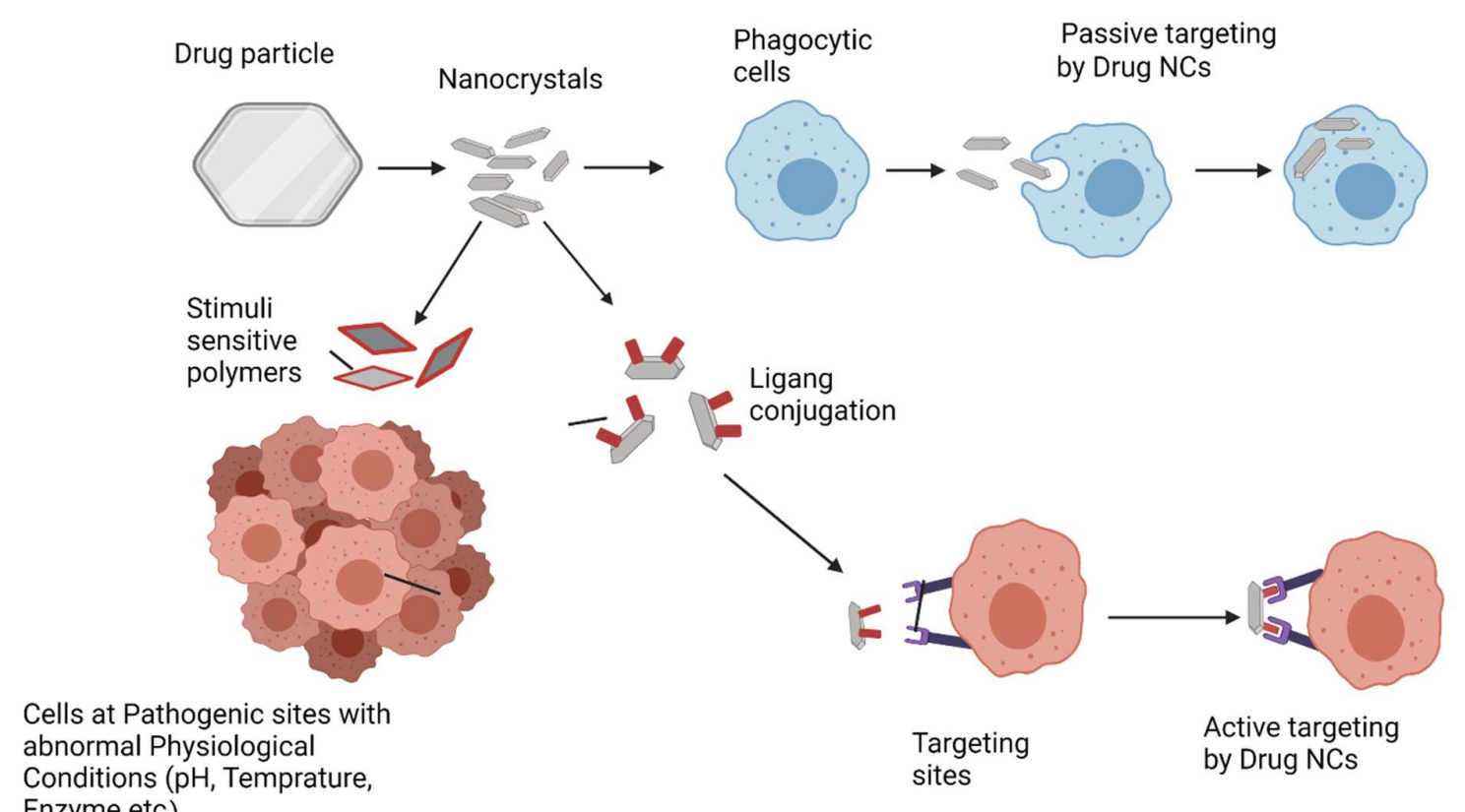

Figure 2. Mechanisms of active and passive targeting to cancer cells by drug nanocrystals (Created by BioRender)

\subsection{Morphology}

The particle size can affect the retention of drug nanocrystals in cells as the drug nanocrystals smaller than $150 \mathrm{~nm}$ show higher solubility and better accumulation in the liver, lungs, and kidneys than drug solution [16]. However, sometimes nanoparticles ranging from 100 to 150 $\mathrm{nm}$ may possess pharmacokinetic behavior like solution [58]. Due to their immediate dissolution in blood, they are usually not recognized by the mononuclear phagocytic system MPS [59]. In comparison, larger nano crystals (300-400 nm or more) exhibit different biodistribution characteristics to solution as they are recognized as foreign agents by MPS, readily phagocytized, and distributed to the liver, spleen, and lungs [60,61]. Due to submicron particle size, they are cleared by MPS after intravenous injection up to hours that is also influenced by stabilizers and method of preparation i.e, bottom-up or top-down techniques [62]. From Phagocytic cells of MPS, hydrophobic therapeutic agents cross phagolysosomal membrane, enters the cytoplasm, and diffuses out due to drug concentration gradient.

Among morphological characteristics, the shape of nanocrystals has a significant effect on their cellular uptake. Rod-shaped drug nanocrystals have better retention and invasiveness in the pathogenic sites than spherical one due to higher aspect ratios [63,64]. This shapedependent cytotoxicity was observed in rod-shaped 10-hydroxycamptothecin and needle-like camptothecin nanocrystals. Differently shaped nanocrystals have been evaluated for the cellular internalization in Human nasopharyngeal epidermal carcinoma KB cells. Confocal microscopy and fluorescence detection also showed higher retention of rod-like nanocrystals in comparison to spherical ones. This higher retention and enhanced permeability of 
chemotherapeutic drug nanocrystals are favorable in passive targeting of tumor cells. The disrupted vasculature, lymphatic drainage system, and defective endothelium of tumor cells enhance drug nanocrystals' uptake.

\subsection{Type of Stabilizer}

In addition to solubility and biodistribution, stabilizers have a potential role in stability of drug nanocrystal prepared by different methods. Particle size reduction induces an increase in surface area and free surface energy may lead to higher magnitude of attractive molecular forces. As a result of these unsatisfied bonding forces at the particle surface they can cohere with each other and result in larger particle size. Further after being lyophilized the drug particles may develop electrostatic forces due to internal friction. The intensity of charges depends on the nature of compounds and the agitation produced. These phenomena hinder the stability of drug nanocrystals and tend to increase the particle size $[65,66]$. To maintain nanocrystals' integrity, steric stabilizers such as nonionic surfactants e.g. polysorbates, vitamin E tocopheryl polyethylene glycol 1000 succinate (TPGS) or sorbitan esters are added to form a thin layer around the drug particles providing mechanical barrier among particles preventing their size increment. Ionic surfactants like sodium dodecyl sulfate (SDS) or cetyltrimethylammonium bromide (CTAB) decrease the magnitude of electrostatic charges. As a result, the drug nanocrystals remain segregated and avoid Ostwald ripening, maintaining the particle size for longer duration of storage in suspension or lyophilized powder. Polymer stabilizers like Polaxamers Pf68, P f127, P f188, and, among celluloses, hydroxypropyl methylcellulose HPMC, are widely used in nano crystallization. Poloxamers, by absorbing on the surface of drug nanocrystals stabilize the nanodrug particles by steric hindrance preventing aggregation. HPMC inhibit the crystal size growth by hydrogen bonding due to high degree of substitution of methoxy and hydroxypropoxy groups $[67,68]$.

The nature of the stabilizer has significant effect on drug transport and targeting by increasing the permeability of drug. They may enhance the influx of drug nanocrystals by opening the tight junctions in cellular membranes, favoring muco-adhesion or binding to cytoplasmic proteins. Therefore, selecting an appropriate stabilizing agent should be critically considered before selecting other parameters for the formulation of drug nanocrystals $[68,69]$. The stabilizer should have an affinity for the crystal surface to avoid unnecessary shedding. However, sometimes stabilizer shedding in response to cellular enzymes or other stimuli is beneficial for the therapeutic effects. For example, TPGS induces PgP inhibition, therefore, it causes a higher uptake of nanocrystals in tumor cells [70]. The effectiveness of TPGS was evaluated by observing higher cytotoxicity of paclitaxel nanocrystals on KB and H460 cell lines compared to free drug and other nanocrystal formulations without TPGS [19]. Similarly, Tween 80 facilitates the cell membrane permeation by P-gp inhibition and drug retention in the cytosol and minimum efflux to extracellular fluid [17]. Drug solution and paclitaxel nanocrystals prepared containing Tween 80 were evaluated for toxicity on MCF-7 and MDAMB cells as mentioned in table 1 . The $\mathrm{IC}_{50}$ of pure drug solution was $12.17( \pm 1.36) \mathrm{nM}$ and 7.02( \pm 1.27$) \mathrm{nM}$ on MCF-7 and MDA-MB cells, respectively. In comparison, paclitaxel nanocrystals with Tween 80 (PTX/NC-T80) was having higher toxicity on [MCF-7 $\mathrm{IC}_{50}$ 7.41 $( \pm 0.96) \mathrm{nM}]$ and [MDA-MB cells and 4.82( \pm 0.32$) \mathrm{nM}]$.

Stimuli-sensitive polymers responding to physiochemical changes such as temperature, $\mathrm{pH}$, ionic concentration, enzymes, and magnetic field have been markedly used in controlled release Drug Delivery Systems. Therefore, they could be effectively used as stabilizers in nanocrystal preparation for drug targeting. For example, the $\mathrm{pH}$ at the tumor cells or tissues is usually lower than that of normal cells, so $\mathrm{pH}$ responsive polymers could be used for targeted 
drug delivery [71]. Hyaluronic acid coated campothecin nanocrystals were exhibiting a $\mathrm{pH}-$ triggered release behavior, where campthecin was released more rapidly, more than $55 \%$ in the mild acidic condition $(\mathrm{pH}=5.5)$ within three hours while it was only $25 \%$ under $\mathrm{pH}=7.4$ PBS [20]. Biobased $\mathrm{CaCO}_{3} /$ doxorubicin nanocrystals were prepared by high-pressure homogenization. They exhibited selective toxicity at $\mathrm{pH} 4.8$ i.e., $96 \%$, without any nonspecific cytotoxicity as nanocrystals were having minimum toxic effects at neutral $\mathrm{pH} 7.4$ [70]. Reactive oxygen species (ROS) sensitive paclitaxel nanocrystals were prepared by wet milling, using a library of 10 redox sensitive amphiphilic block copolymers. ROS-responsive biodegradable polymeric stabilizers were prepared by post-polymerization modification via the thiol-yne reaction [72]. The hydrophobic thiols had an affinity for hydrophobic surface of oxidation-sensitive paclitaxel nanocrystals. This study concluded that site-specific shedding of stabilizers could be used in drug targeting by cellular uptake. Therefore, other stimuli-sensitive polymers could target the drug to specific infectious regions for localized effects without affecting the healthy cells e.g. poloxamers including Pluronic F68 and Pluronic F127, Poly(ethylene glycol)-poly(3-caprolactone)-poly(ethylene glycol) (PCEC).

\subsection{Ligand attachment}

The nanocrystal surface modification by stabilizers is essential for internalizing tumor cells and avoiding rapid clearance by MPS. In addition to the stabilizer layer, the presence of ligands is beneficial for site-specific targeting of the drugs to tumor cells. The ligand molecules can bind specific sites at the receptors of malignant cells, usually without affecting healthy sites. Numerous receptor molecules present on cells may serve as docking sites for anticancer drug targeting like folate receptors, transferrin receptors, sigma receptors [73], bombesin receptors [74], fibroblast growth factor receptors, follicle stimulating hormone receptors etc. Understanding the appropriate interactions between nanocrystals and cell membrane receptors is significant in therapeutical applications [75-78]. Several factors like ligand molecule length and density affect the equilibrium in receptor-mediated endocytosis. Higher density and rigidity increase the uniform distribution of ligand molecules on drug particle surface. The ligand length is proportional to binding affinity on cell membranes, but it is usually harder for phagocytic cells to engulf the drug nanocrystals with longer ligands. In addition, as previously discussed, particle size, shape, and surface texture of drug nanoparticles can also affect the receptor-mediated endocytosis [79-83].

Some of the commonly used ligands, ligand conjugated chemotherapeutic agents and their enhanced permeation in cancer cell models and cell lines are presented in table 2 and discussed afterwards. Among all cell receptors, the folate receptors are commonly overexpressed on plasma membrane of most cancer cells, including lungs, endometrial, breast, ovarian, and kidney cancers [84-89]. Folate receptors mediate the cellular uptake of folic acid, also known as vitamin B9, an essential vitamin for cell proliferation. Therefore, folate receptors are higher in rapidly dividing cancerous cells. However, the folic acid is also taken by healthy cells via proton-coupled folate transporter and reduced folate carrier system [90-93]. Folic acid has been extensively studied for its higher cell penetration, lower immunogenic potential, and more straightforward modification. The effect of folic acid conjugated cellulose nanocrystals was determined on folate receptor-positive cells, KB cells and human breast cells (MDA-MB468). It showed selective targeting and higher internalization in the cell lines. This proves them as potential diagnostic imaging agents for tumor detection at the early stages [94]. Elongated folic acid modified cellulose nanocrystals and unmodified cellulose crystals were evaluated for their cellular uptake to human brain tumor cells DBTRG-05MG, H4 and C6 rat brain tumor cells. Folate receptor expression of the cells was verified by immunofluorescence staining. The modified nanocrystals exhibited higher cellular binding in DBTRG-05MG, H4, and C6 cells. 
They were1452, 975, and 46 times higher, respectively, than nanocrystals without folic acid. The cellular internalization was by caveole- mediated endocytosis in DBTRG-05MG and C6 cells and in H4 cells via clathrin-mediated endocytosis [95, 96].

Table 2. Ligand conjugated targeting of drugs to tumor cells and cell lines

\begin{tabular}{|l|l|l|c|}
\hline Chemotherapeutic agents & Ligands & Tumor cells/ cell lines & References \\
\hline Calcium fluorapatite & Folic acid & HepG2 and MCF-7 & {$[97]$} \\
\hline Campothecin and paclitaxel & Folic acid & $\begin{array}{l}\text { Human lung cancer and } \\
\text { murine breast cancers }\end{array}$ & {$[99]$} \\
\hline $\begin{array}{l}\text { Cadmium selenide/Cadmium } \\
\text { Sulphide (CdSe/CdS) }\end{array}$ & $\begin{array}{l}\text { Folic acid and } \\
\text { Biotin }\end{array}$ & HeLa, KB cells & {$[100]$} \\
\hline Paclitaxel & $\begin{array}{l}\text { transferrin, } \\
\text { hyaluronic acid }\end{array}$ & MCF-7 & {$[20]$} \\
\hline Doxorubicin & Transferrin & HeLa & {$[23]$} \\
\hline Cadmium chalcogenide & Transferrin & H460 & {$[107]$} \\
\hline
\end{tabular}

Folic acid-conjugated bioluminescent calcium fluorapatite nanocrystals for targeted cancer cell fluorescence imaging were evaluated for internalization in positive folate receptor cells. The nanocrystals were biocompatible and readily dispersible in water [97]. Furthermore, by grafting nanocrystal's surface with folic acid, they had highly sensitive recognition of targeted sites. This was evaluated by confocal laser scanning microscopy regarding fluorescence imaging of nanoprobes for folate receptor in tumor cells i.e., HepG2 and MCF-7 cells. HepG2 are human hepatocellular carcinoma cells with high growth rate and overexpression of folate compared to MCF-7 cell lines with human breast adenocarcinoma cells with a slower growth rate and negligible expression of folate receptors [98].

A nanocrystal formulation of hydrophobic anticancer drugs paclitaxel and camptothecin was developed using Pluronic F127 polymer as a stabilizer by three-phase nanoparticle engineering technology, including amorphous precipitation, amorphous aggregation, and nanocrystal stabilization [99]. The nanocrystals encapsulated over $99 \%$ of the drug with a high ratio of drug to excipient. Antitumor activity was evaluated in two tumor models, human lung and murine breast cancers, resulting in significant inhibition of tumor growth by intravenous and oral administration. The nanocrystals were further modified for targeted delivery of paclitaxel by conjugating a folate ligand to the outer stabilizer layer of F127. The folic acid conjugated nanocrystals were showing low cell viability. The paclitaxel nanocrystals induced maximum toxicity at $10 \%$ concentration of F127-folate.

Biotin and folic acid functionalized Cadmium selenide/Cadmium Sulphide (CdSe/CdS) nanocrystals were formulated by new seeded-type growth [100]. Biotin-conjugated nanorods were evaluated by incubating in HeLa cells and exploited as an alternative class of fluorescent molecular probes for cell and tissue imaging. Other nanocrystals conjugated with folic acid 
were studied for selective targeting on human nasopharyngeal epidermal carcinoma (KB) cells, showed the potential of these materials for targeting and imaging specific tumor cells [101].

In addition to folic acid, the surface of nano drug delivery systems could be modified by different agents like proteins, polysaccharides, peptides, aspartame, and small molecules that enhance the antitumor effect [102 - 104]. Among all, transferrin has broad utility as surfacemodifier of nanocrystals for targeting tumor cells, which could be due to higher iron demand of cancer cells and overexpression of transferrin-receptor. Therefore, transferrin-receptor mediated endocytosis is an efficiently involved cellular uptake pathway for delivering anticancer agents $[105,106]$. Transferrin conjugated nanocrystals have enhanced internalization into various cell lines like A549, MCF-7, MDA-MB-231 cells, HeLa cells, Caco-2 cells human H460 cell line etc. For example, transferrin-modified paclitaxel nanocrystals with TPGS1000 as the stabilizer were prepared successfully for oral administration with improved intestinal absorption and resulted in higher antitumor effect [19]. Because hyaluronic acid could specially react with the overexpressed CD44 receptors on MDA-MB-231cell line. In a study mentioned in table 1, hyaluronic acid-modified paclitaxel nanocrystals were prepared and evaluated for cytotoxicity and cellular uptake in MDA-MB231 cells. Nanocrystals were having 30.8\% cellular uptake whereas pure drug solution exhibited $15 \%$ [20]. In another study, different types of paclitaxel nanocrystals were prepared by the nano-precipitation method. The surface of nanocrystals was modified with transferrin, hyaluronic acid and compared with unmodified paclitaxel nanocrystals. All nanocrystals modified and unmodified had a mean particle size of $230-340 \mathrm{~nm}$. The modified nanocrystals were showing higher cellular uptake in MCF-7 cells. The cell growth inhibition in MCF-7 cells was significantly higher by modified nanocrystals than unmodified nanocrystals and pure drug. On the other hand, transferrin and hyaluronic acid-modified nanocrystals were safer in normal cells i.e., HaCaT cells with cell inhibition 11 to $12 \%$, while in unmodified paclitaxel nanocrystals and pure drug, the cell inhibition was $17 \%$ and $23 \%$, respectively [18]. Transferrin modified docetaxel nanocrystals after incubation of 24 hours in A549 cells were possessing higher cytotoxicity $(66.9 \% \pm 3.8 \%)$ than unmodified docetaxel nanocrystals $(55.5 \% \pm 6.1 \%)$ and pure drug $(15.5 \% \pm 5.7 \%)$ at a docetaxel concentration of $100 \mu \mathrm{g} / \mathrm{ml}[23]$.

Stronger binding approach of ligands/biomolecules to drug crystal surface is needed to improve cellular targeting and nanocrystal uptake. This formulation parameter of nanocrystal modification is critical for biomedical applications. A facet engineering approach was adopted for significantly enhancing transferrin binding to cadmium chalcogenide nanocrystals. Their cellular uptake was evaluated by confocal microscopy on HeLa cells [107]. The stronger binding between transferrin and nanocrystal resulted in greater uptake of these proteinnanocrystal conjugates into HeLa cells. Transferrin binding was obtained by inner-sphere thiol complexation, evaluated by competitive adsorption experiments and theoretical calculations. High thiol content contributes to the higher binding of transferrin to nanocrystals possessing soft metals by metal-thiol complexation. Further, molecular dynamics simulation revealed that facet-dependent transferrin modification occurred by the differential affinity of crystal facets to the monomolecular layer of water molecules, which hinders access to exposed facets [108]. Chemical complexation could be widely adopted for adsorption of ligand macromolecules onto metal-containing nanomaterials, could be extended to different biocompatible nanocrystals preferably containing soft metals (e.g., $\mathrm{Au}, \mathrm{Ag}, \mathrm{Pt}, \mathrm{Pd}$, and $\mathrm{Zn}$ ) having a strong affinity to form coordination bonds with thiol-containing ligands $[109,110]$.

Transferrin conjugated to alloyed quaternary nanocrystals was evaluated to have high recognition of tumor sites possessing higher cellular uptake in tumor cells and low toxicity to healthy cells. Stable, biocompatible nanoconjugate of transferrin anchored to Ag- In- Zn-S 
quantum dots on the surface of doxorubicin nanocrystals. The drug nanoconjugate exhibited a concentration-dependent cytotoxic effect on tumor cells H460 cell line (human non-small cell lung carcinoma) [111].

\section{Conclusion}

Advancement in pharmaceutical technology by formulating colloidal drug delivery systems could lead to a revolution in dosage form design, drug administration, and treatment of lifethreatening diseases. Among all nano drug delivery systems, nanocrystals are pure drug particles with a nominal quantity of stabilizing agents that could be easily scaled up for industrial manufacturing. Nano crystallization overcomes or minimize bioavailability problems of poorly soluble drugs. Furthermore, drug nanocrystals have the advantage of delivering the therapeutic agents in high concentrations to the cancer tissues due to their morphological characteristics, stabilizers, and ligand attachment. Nanocrystals could be used for site specific targeted drug delivery with higher cell internalization, preventing normal body tissues and minimize drug related side effects.

\section{References}

1) Muller, R.H.; Keck, C.M. Challenges and solutions for the delivery of biotech drugs-a review of drug nanocrystal technology and lipid nanoparticles. J. Biotechnol. 2004,113, 151-70.

2) Bertrand, N.; Leroux, J.C. The journey of a drug carrier in the body: an anatomophysiological perspective. J.Contr Rel. 2011, 161 : 152-63.

3) Zhang, L.; Hou, S.; Mao, S.; Wei, D.; Song, X.; Lu, Y. Uptake of folate-conjugated albumin nanoparticles to the SKOV3 cells. Int. J. Pharm. 2004, 287, 155-62.

4) Sudimack, J.; Lee, R.J. Targeted drug delivery via the folate receptor. Adv. Drug. Deliv. Rev. 2000, 41, 147-62.

5) Vader, P. J van der Aa L, Storm G. M Schiffelers R, FJ Engbersen J. Polymeric carrier systems for siRNA delivery. CTMCCL. 2012, 12, 108-190.

6) Veiseh, F.M.; Kievit, R.G. Ellenbogen, M. Zhang, Cancer cell invasion: treatment and monitoring opportunities in nanomedicine, Adv. Drug Deliv. Rev. 2011, 582-596.

7) Kreuter, J.; Petrov, V.E.; Kharkevich, D.A.; Alyautdin, R.N.; Influence of the type of surfactant on the analgesic effects induced by the peptide dalargin after its delivery across the blood-brain barrier using surfactant-coated nanoparticles. J. Contr. Rel. 1997, 49, 81-7.

8) Gao, L.; Liu, G.; Ma, J.; Wang, X.; Zhou, L.; Li, X. Drug nanocrystals: in vivo performances. J. Control. Rel. 2012, 160, 418-30.

9) Rabinow, B.E. Nanosuspensions in drug delivery. Nat. Rev. Drug Discov. 2004, 3,78596.

10) Joshi, K.; Chandra, A.; Jain, K.; Talegaonkar, S. Nanocrystalization: an emerging technology to enhance the bioavailability of poorly soluble drugs. Pharm. Nanotechnol. 2019, 7, 259-78.

11) Chang, T.L.; Zhan, H.; Liang, D.; Liang, J.F. Nanocrystal technology for drug formulation and delivery. Front. Chem. Sci. \& Eng. 2015 9, 1-4.

12) Liu, T.; Yu, X.; Yin, H.; Möschwitzer, J.P. Advanced modification of drug nanocrystals by using novel fabrication and downstream approaches for tailor-made drug delivery. Drug Deliv. 2019, 26, 1092-103. 
13) Joshi, K.; Chandra, A.; Jain, K.; Talegaonkar, S. Nanocrystalization: an emerging technology to enhance the bioavailability of poorly soluble drugs. Pharm. Nanotechnol, 2019, 7, 259-78.

14) Zhao, J.; Liu, Y.; Wang, L.; Zhou, Y.; Du, J.; Wang, Y. Functional and modified nanocrystals technology for target drug delivery. J Nanosci Nanotechnol 2018, 18, 5207-21.

15) Joshi, K.; Chandra, A.; Jain, K.; Talegaonkar, S. Nanocrystalization: an emerging technology to enhance the bioavailability of poorly soluble drugs. Pharm. Nanotechnol. 2019, 7, 259-78..

16) Ganta, S.; Paxton, J.W.; Baguley, B.C.; Garg, S. Formulation and pharmacokinetic evaluation of an asulacrine nanocrystalline suspension for intravenous delivery. Int. J. Pharm. Sci. 2009, 367, 179-86.

17) Sharma, S.; Verma, A .; Teja, B.V.; Shukla, P.; Mishra, P.R. Development of stabilized paclitaxel nanocrystals: in-vitro and in-vivo efficacy studies. Eur. J. Pharm. Sci. 2015, 69:51-60.

18) Sohn, J.S.; Yoon, D.S.; Sohn, J.Y.; Park, J.S.; Choi, J.S. Development and evaluation of targeting ligands surface modified paclitaxel nanocrystals. Mater. Sci. \& Eng. 2017 Mar 1; 72:228-37.

19) Liu, Y.; Huang, L.; Liu, F. Paclitaxel nanocrystals for overcoming multidrug resistance in cancer. Mol. Pharm. 2010, 7, 863-9.

20) Wang, J.; Muhammad, N.; Li, T.; Wang, H.; Liu, Y.; Liu, B.; Zhan, H. Hyaluronic acidcoated camptothecin nanocrystals for targeted drug delivery to enhance anticancer efficacy. Mol. Pharm. 2020 17, 2411-25.

21) Zhan, H.; Liang, J.F. Extreme activity of drug nanocrystals coated with a layer of noncovalent polymers from self-assembled boric acid. Sci. Rep. 2016, 6, 1-11.

22) Choi, J.S.; Park, J.S. Surface modification of docetaxel nanocrystals with HER2 antibody to enhance cell growth inhibition in breast cancer cells. COLLOID SURFACE B. 2017, 159, 139-50.

23) Choi, J.S.; Park, J.S. Development of docetaxel nanocrystals surface modified with transferrin for tumor targeting. Drug. Des. Devel. Ther. 2017, 11:17.

24) Martin, B.; Seguin, J.; Annereau, M.; Fleury, T.; Lai-Kuen, R.; Neri, G, Lam, A.; Bally, M.; Mignet, N.; Corvis, Y. Preparation of parenteral nanocrystal suspensions of etoposide from the excipient free dry state of the drug to enhance in vivo antitumoral properties. Sci. Rep. 2020, 10, 1-3.

25) Kong, F.H.; Ye, Q.F.; Miao, X.Y.; Liu, X.; Huang, S.Q.; Xiong, L.; Wen, Y.; Zhang, Z.J. Current status of sorafenib nanoparticle delivery systems in the treatment of hepatocellular carcinoma. Theranostics. 2021, 11, 5464.

26) Sheng, H.; Zhang, Y.; Nai, J.; Wang, S.; Dai. M.; Lin, G.; Zhu, L.; Zhang, Q. Preparation of oridonin nanocrystals and study of their endocytosis and transcytosis behaviours on MDCK polarized epithelial cells. Pharm. Biol.2020,58, 518-27.

27) Liang, P.; Wu, H.; Zhang, Z.; Jiang, S.; Lv, H. Preparation and characterization of parthenolide nanocrystals for enhancing therapeutic effects of sorafenib against advanced hepatocellular carcinoma. Int. J. Pharm.. 2020 15;583:119375.

28) Lu, Y.; Li, Y.; Wu, W. Injected nanocrystals for targeted drug delivery. Acta Pharmaceutica Sinica B. 2016, 6, 106-13.

29) Gao, L.; Liu, G.; Ma, J.; Wang, X.; Zhou, L.; Li, X. Drug nanocrystals: in vivo performances. J. Control. Release. 2012, 160, 418-30.

30) Gao, L.; Zhang, D.; Chen, M.; Duan, C.; Dai, W.; Jia, L.; Zhao, W. Studies on pharmacokinetics and tissue distribution of oridonin nanosuspensions. Int. J. Pharm. 2008, 355, 321-7. 
31) Malamatari, M.; Taylor, K.M.; Malamataris, S.; Douroumis, D.; Kachrimanis, K. Pharmaceutical nanocrystals: production by wet milling and applications. Drug Discov. Today. 2018, 23, 534-47.

32) Peltonen L, Hirvonen J. Pharmaceutical nanocrystals by nanomilling: critical process parameters, particle fracturing and stabilization methods. J. Pharm. Pharmacol. 2010, $62,1569-79$.

33) Merisko-Liversidge, E.; Liversidge, G.; Cooper, E.R. Nanosizing: a formulation approach for poorly-water-soluble compounds. Eur. J. Pharm. Sci. 2003, 18, 113-120.

34) Parmentier, J.; Widzinski, M.; Tan, E. H.; Gokkhale, R.; Chin, W. L. A brief literature and patent review of nanosuspensions to a final drug product. J. Pharm. Sci. 2014, 103, 2980-2999.

35) Muller, R.H.; Keck, C.M. Drug nanocrystals of poorly soluble drugs produced by high pressure homogenization. Eur. J. Pharm. Biopharm. 2006, 62, 3-16.

36) Gohla, S.; Keck, C. M.; Muller, R. H. State of the art of nanocrystals-Special features, production, nanotoxicology aspects and intracellular delivery. Eur. J. Pharm. Biopharm. 2011, 78, 1-9

37) Chang, T.L.; Zhan, H.; Liang, D.; Liang, J.F. Nanocrystal technology for drug formulation and delivery. Front. Chem. Sci. \& Eng. 2015, 9, 1-4.

38) Sharma, P.; Zujovic, Z.D.; Bowmaker, G.A.; Denny, W.A.; Garg, S. Evaluation of a crystalline nanosuspension: polymorphism, process induced transformation and in vivo studies. Int. J. Pharm. 2011, 408,138-51.

39) Liu, T.; Müller, R.H.; Möschwitzer, J.P. Production of drug nanosuspensions: effect of drug physical properties on nanosizing efficiency. Drug Dev. Ind. Pharm. 2018, 44, 233-42.

40) Cerdeira, A.M.; Gander, B.; Mazzotti, M. Role of milling parameters and particle stabilization on nanogrinding of drug substances of similar mechanical properties. Chem. Eng. Technol. 2011, 34,1427-38.

41) Salazar J, Müller RH, Möschwitzer JP. Application of the combinative particle size reduction technology $\mathrm{H} 42$ to produce fast dissolving glibenclamide tablets. Eur. J. Pharm. Sci. 2013, 49, 565-77.

42) Liu, T.; Müller, R.H.; Möschwitzer, J.P. Systematical investigation of a combinative particle size reduction technology for production of resveratrol nanosuspensions. AAPS PharmSciTech, 2017, 18, 1683-1691.

43) Moorthi, C.; Kathiresan, K. Fabrication of highly stable sonication assisted curcumin nanocrystals by nanoprecipitation method. Drug Invent. Today. 2013, 5, 66-9.

44) List, M.; Sucker, H. Pharmaceutical colloidal hydrosols for injection. GB patent. 1988;2200048.

45) Soliman, K.A.; Ibrahim, H.K.; Ghorab, M.M. Effects of different combinations of nanocrystallization technologies on avanafil nanoparticles: in vitro, in vivo and stability evaluation. Inter. J. Pharm. 2017, 517, 148-56.

46) Bosselmann, S.; Nagao, M.; Chow, K.T.; Williams, R.O. Influence of formulation and processing variables on properties of itraconazole nanoparticles made by advanced evaporative precipitation into aqueous solution. AAPS PharmSciTech. 2012, 13, 94960.

47) de Waard, H.; Frijlink, H.W.; Hinrichs, W.L. Bottom-up preparation techniques for nanocrystals of lipophilic drugs. Pharm. Res.2011, 28, 1220-3.

48) Montes, A.; Gordillo, M.D.; Pereyra, C.; de la Ossa, E.M. Particles formation using supercritical fluids. Intech Open; 2011. 
49) Spitzer, D.; Pichot, V.; Pessina F.; Schnell, F.; Klaumünzer, M.; Blas, L. Continuous and reactive nanocrystallization: New concepts and processes for dual-use advances. CR Chim.2017, 20, 339-45.

50) Möschwitzer. J.; Müller, R.H. New method for the effective production of ultrafine drug nanocrystals. J. Nanosci. Nanotechnol. 2006, 6, 3145-53.

51) Chang, T.L.; Zhan, H.; Liang, D.; Liang, J.F. Nanocrystal technology for drug formulation and delivery. Front. Chem. Sci. Eng. 2015, 9, 1-4.

52) Salazar, J.; Müller, R.H.; Möschwitzer, J.P. Combinative particle size reduction technologies for the production of drug nanocrystals. Int. J. Pharm.2014, 2014, 1-14.

53) Shegokar, R.; Müller, R.H. Nanocrystals: industrially feasible multifunctional formulation technology for poorly soluble actives. Int. J. Pharm. 2010 399,129-39.

54) Verma, S.; Gokhale, R.; Burgess, D.J. A comparative study of top-down and bottomup approaches for the preparation of micro/nanosuspensions. Int. J. Pharm. 2009 380, 216-22.

55) Müller, R.H.; Keck, C.M. Second generation of drug nanocrystals for delivery of poorly soluble drugs: smart Crystal technology. Eur. J. Pharm. Sci. 2008, 1, 20-1.

56) Haynes, D.H. Phospholipid-coated microcrystals: Injectable formulations of water insoluble drugs. US Patent, 5091187, 1992.

57) Liu. G.; Zhang, D.; Jiao, Y.; Guo, H.; Zheng, D.; Jia, L.; Duan, C.; Liu, Y.; Tian, X.; Shen, J.; Li, C. In vitro and in vivo evaluation of riccardin D nanosuspensions with different particle size. COLLOID SURFACE B. 2013, 102, 620-6.

58) Lu, Y.; Li, Y.; Wu, W. Injected nanocrystals for targeted drug delivery. Acta Pharm. Sin. B. 2016, 6, 106-13.

59) Gao, L.; Liu, G.; Ma, J.; Wang, X.; Zhou, L.; Li, X. Drug nanocrystals: in vivo performances. J. Control. Release. 2012, 160, 418-30.

60) Gao, L.; Zhang, D.; Chen, M.; Duan, C.; Dai, W.; Jia, L.; Zhao, W. Studies on pharmacokinetics and tissue distribution of oridonin nanosuspensions. Int. J. Pharm. 2008, 355, 321-7.

61) Liu, G.; Zhang, D.; Jiao, Y.; Guo, H.; Zheng, D.; Jia, L.; Duan, C.; Liu, Y.; Tian, X.; Shen, J.; Li, C. In vitro and in vivo evaluation of riccardin D nanosuspensions with different particle size. COLLOID SURFACE B. 2013, 102, 620-6.

62) Liu, T.; Yu, X.; Yin, H.; Möschwitzer, J.P. Advanced modification of drug nanocrystals by using novel fabrication and downstream approaches for tailor-made drug delivery. Drug Deliv. 2019, 26, 1092-103.

63) Li, W.; Zhang, X.; Hao, X.; Jie, J.; Tian, B.; Zhang, X. Shape design of high drug payload nanoparticles for more effective cancer therapy. Chem. Commun. 2013, 49, 10989-91.

64) Zhang, H.; Hollis, C.P.; Zhang, Q.; Li, T. Preparation and antitumor study of camptothecin nanocrystals. Int. J. Pharm. 2011, 415, 293-300.

65) Lachman, L.; Lieberman, H.A.; Kanig, J.L. The theory and practice of industrial pharmacy. Philadelphia: Lea \& Febiger; 1976.

66) Beirowski, J.; Inghelbrecht, S.; Arien, A.; Gieseler, H. Freeze-drying of nanosuspensions, 1: freezing rate versus formulation design as critical factors to preserve the original particle size distribution. J. Pharm. Sci. 2011 100, 1958-68.

67) Goodman, E.D. Colloidal Design of Active, Selective, and Stable Catalysts for Methane Utilization. Stanford University; 2020.

68) Tuomela, A.; Hirvonen, J.; Peltonen, L. Stabilizing agents for drug nanocrystals: effect on bioavailability. Pharmaceutics. 2016, 8:16. 
69) Peltonen, L.; Hirvonen, J. Pharmaceutical nanocrystals by nanomilling: critical process parameters, particle fracturing and stabilization methods. J. Pharm. Pharmacol.. 2010 Nov;62(11):1569-79.

70) Dintaman, J.M.; Silverman, J.A. Inhibition of P-glycoprotein by D- $\alpha$-tocopheryl polyethylene glycol 1000 succinate (TPGS). Pharm. Res.1999, 10, 1550-6.

71) Tang, H.; Zhao, W.; Yu, J.; Li, Y.; Zhao, C. Recent development of pH-responsive polymers for cancer nanomedicine. Molecules. 2019, 24,4.

72) Fuhrmann, K.; Połomska, A.; Aeberli, C.; Castagner B, Gauthier MA, Leroux JC. Modular design of redox-responsive stabilizers for nanocrystals. ACS Nano. 2013,7, 8243-50.

73) Georgiadis, M.O.; Karoutzou, O.; Foscolos, A.S.; Papanastasiou, I. Sigma receptor $(\sigma \mathrm{R})$ ligands with antiproliferative and anticancer activity. Molecules. 2017, 22,1408.

74) Pooja, D.; Gunukula, A.; Gupta, N.; Adams, D.J.; Kulhari, H. Bombesin receptors as potential targets for anticancer drug delivery and imaging. INT J BIOCHEM CELL B. 2019, 114,105567.

75) Nel, A.E.; Mädler, L.; Velegol, D.; Xia, T.; Hoek, E.M, Somasundaran, P.; Klaessig, F.; Castranova, V.; Thompson, M. Understanding biophysicochemical interactions at the nano-bio interface. Nat. Mater.2009, 8, 543-57.

76) Mitragotri, S.; Lahann, J. Physical approaches to biomaterial design. Nat. Mater.2009, 8, 15-23.

77) Verma, A.; Stellacci, F. Effect of surface properties on nanoparticle-cell interactions. small. $2010,6,12-21$.

78) Leroueil, P.R.; Hong, S.; Mecke, A.; Baker, Jr JR, Orr BG, Banaszak Holl M.M. Nanoparticle interaction with biological membranes: does nanotechnology present a Janus face?. Acc. Chem. Res.. 2007, 40, 335-42.

79) Ding, H.M, Ma, Y.Q. Role of physicochemical properties of coating ligands in receptor-mediated endocytosis of nanoparticles. Biomaterials. 2012, 33, 5798-802.

80) Gratton, S.E.; Ropp, P.A.; Pohlhaus, P.D.; Luft, J.C.; Madden, V.J.; Napier, M.E.; DeSimone, J.M. The effect of particle design on cellular internalization pathways. PNAS. 2008, 105, 11613-8.

81) Chithrani, B.D.; Ghazani, A.A.; Chan, W.C. Determining the size and shape dependence of gold nanoparticle uptake into mammalian cells. Nano Lett. 2006, 6, 6628 .

82) Gao, H.; Shi, W.; Freund, L.B. Mechanics of receptor-mediated endocytosis. PNAS. 2005, 102, 9469-74.

83) Vácha, R.; Martinez-Veracoechea, F.J.; Frenkel, D. Receptor-mediated endocytosis of nanoparticles of various shapes. Nano Lett.. 2011, 11, 5391-5.

84) Verma, A.; Uzun, O.; Hu, Y.; Hu, Y.; Han, H.S. Surface-structure-regulated cellmembrane penetration by monolayer-protected nanoparticles. Nat. Mater.2008, 7, 58895.

85) Dainty, L.A.; Risinger, J.I.; Morrison, C.; Maxwell, G.L. Overexpression of folate binding protein and mesothelin are associated with uterine serous carcinoma. Gynecol. Oncol. 2007, 105, 563-70.

86) WL, H.L.; Christianson, T.J.H.; Varghese, B.; Hillman, D.; Oberg, A.L.; Low, P.S. Int. J. Cancer. 2007, 121, 938-42.

87) Toffoli, G.; Cernigoi, C.; Russo, A.; Gallo, A.; Bagnoli, M.; Boiocchi, M. Overexpression of folate binding protein in ovarian cancers. Int. J. Cancer. 1997 Apr 22;74(2):193-8.

88) Weitman, S.D.; Frazier, K.M.; Kamen, B.A. The folate receptor in central nervous system malignancies of childhood. J. Neuro-Oncol. 1994, 21, 107-12. 
89) Weitman, S.D.; Weinberg, A.G.; Coney, L.R.; Zurawski, V.R.; Jennings, D.S.; Kamen, B.A. Cellular localization of the folate receptor: potential role in drug toxicity and folate homeostasis. Cancer Res.1992, 52, 6708-11.

90) Weitman, S.D.; Lark, R.H.; Coney, L.R.; Fort, D.W.; Frasca, V.; Zurawski ,V.R.; Kamen, B.A. Distribution of the folate receptor GP38 in normal and malignant cell lines and tissues. Cancer Res.. 1992, 52, 3396-401.

91) Hutson, J.R.; Stade, B.; Lehotay, D.C.; Collier, C.P.; Kapur, B.M. Folic acid transport to the human fetus is decreased in pregnancies with chronic alcohol exposure. PloS one. 2012, 7, e38057.

92) Salazar, M.D.; Ratnam, M. The folate receptor: what does it promise in tissue-targeted therapeutics?. Cancer Metastasis Rev. 2007, 26, 141-52.

93) Dong, S.; Cho, H.J.; Lee, Y.W.; Roman, M. Synthesis and cellular uptake of folic acidconjugated cellulose nanocrystals for cancer targeting. Biomacromolecules. 2014, 15, 1560-7.

94) Bittleman, K.R.; Dong, S.; Roman, M.; Lee, Y.W. Folic acid-conjugated cellulose nanocrystals show high folate-receptor binding affinity and uptake by KB and breast cancer cells. ACS omega. 2018,3, 13952-9.

95) Talekar, M.; Ganta, S.; Amiji, M.; Jamieson, S.; Kendall. J.; Denny, W.A.; Garg, S. Development of PIK-75 nanosuspension formulation with enhanced delivery efficiency and cytotoxicity for targeted anticancer therapy. Inter. J. Pharm. 2013 450, 278-89.

96) Dong, S.; Cho. H.J.; Lee, Y.W.; Roman, M. Synthesis and cellular uptake of folic acidconjugated cellulose nanocrystals for cancer targeting. Biomacromolecules. 2014, 15, 1560-7.

97) Xu, J.; Shen, X.; Jia, L.; Xu, Z.; Zhou, T.; Li, X. Facile synthesis of folic acidconjugated fluorapatite nanocrystals for targeted cancer cell fluorescence imaging. Mater. Lett. 2017,203, 37-41.

98) Monteiro, C.A.; Oliveira, A.D.; Silva, R.C.; Lima, R.R.; Souto, F.O.; Baratti, M.O.; Carvalho, H.F.; Fontes, A. Evaluating internalization and recycling of folate receptors in breast cancer cells using quantum dots. J. Photochem. Photobiol. B. 2020, 209, 111918.

99) Liu, F.; Park, J.Y.; Zhang, Y., Conwell, C., Liu, Y., Bathula, S.R. and Huang, L., Targeted cancer therapy with novel high drug-loading nanocrystals. J. Pharm. Sci. 2010, 99, 3542-3551.

100) Yang, X.; Yang, S.; Chai, H.; Yang, Z.; Lee, R.J.; Liao, W.; Teng L. A novel isoquinoline derivative anticancer agent and its targeted delivery to tumor cells using transferrin-conjugated liposomes. PLoS One. 2015, 10, 0136649.

101) Quarta, A.; Ragusa, A.; Deka, S.; Tortiglione, C.; Tino, A.; Cingolani, R.; Pellegrino, T. Bioconjugation of rod-shaped fluorescent nanocrystals for efficient targeted cell labeling. Langmuir. 2009, 25, 12614-22.

102) Almalik, A.; Donno, R.; Cadman, C.J.; Cellesi, F.; Day, P.J.; Tirelli, N. Hyaluronic acid-coated chitosan nanoparticles: Molecular weight-dependent effects on morphology and hyaluronic acid presentation. J. Control. Release. 2013, 172, 1142-50.

103) Surassmo, S.; Saengkrit, N.; Ruktanonchai, U.R.; Suktham, K.; Woramongkolchai, N.; Wutikhun, T.; Puttipipatkhachorn, S. Surface modification of PLGA nanoparticles by carbopol to enhance mucoadhesion and cell internalization. COLLOID SURFACE B. 2015, 130, 229-36.

104) Anisha, B.S.; Biswas, R.; Chennazhi, K.P.; Jayakumar, R. Chitosan-hyaluronic acid/nano silver composite sponges for drug resistant bacteria infected diabetic wounds. Int. J. Biol. Macromol. 2013, 62, 310-20. 
105) Pang, Z.; Gao, H.; Yu, Y.; Chen, J.; Guo, L.; Ren, J.; Wen, Z.; Su, J.; Jiang, X. Brain delivery and cellular internalization mechanisms for transferrin conjugated biodegradable polymersomes. Inter. J. Pharm. 2011, 415, 284-92.

106) Han, S.; Li, X.; Zhou, C.; Hu, X.; Zhou, Y.; Jin, Y.; Liu, Q.; Wang, L.; Li, X.; Liu, Y. Further Enhancement in Intestinal Absorption of Paclitaxel by Using TransferrinModified Paclitaxel Nanocrystals. ACS Appl. Bio Mater. 2020 Jun, 3, 4684-95.

107) Qi, Y.; Zhang, T.; Jing, C.; Liu, S.; Zhang, C.; Alvarez, P.J.; Chen, W. Nanocrystal facet modulation to enhance transferrin binding and cellular delivery. Nat. Commun. 2020, 11, 1-0.

108) Penna, M.J.; Mijajlovic, M.; Biggs, M.J. Molecular-level understanding of protein adsorption at the interface between water and a strongly interacting uncharged solid surface. J. Am. Chem. Soc. 2014, 136, 5323-31.

109) Lau, B.L.; Hsu-Kim, H. Precipitation and growth of zinc sulfide nanoparticles in the presence of thiol-containing natural organic ligands. Environ. Sci. Technol. 2008, 42, 7236-41.

110) Wang, L.; Li. J.; Pan, J.; Jiang, X. Revealing the binding structure of the protein corona on gold nanorods using synchrotron radiation-based techniques: understanding the reduced damage in cell membranes. J. Am. Chem. Soc. 2013, 135, 17359-68.

111) Matysiak-Brynda, E.; Bujak, P.; Augustin, E.; Kowalczyk, A.; Mazerska, Z.; Pron, A.; Nowicka, A.M. Stable nanoconjugates of transferrin with alloyed quaternary nanocrystals $\mathrm{Ag}-\mathrm{In}-\mathrm{Zn}-\mathrm{S}$ as a biological entity for tumor recognition. Nanoscale. 2018, 10,1286-96. 